\title{
On the Convergence of Relaxation Schemes to Couple Phasor-Mode and Electromagnetic Transients Simulations
}

\author{
Frédéric J. Plumier ${ }^{a}$ \\ f.plumier@ulg.ac.be
}

\author{
Christophe Geuzaine ${ }^{a}$ \\ cgeuzaine@ulg.ac.be
}

\author{
Thierry Van Cutsem ${ }^{b}$ \\ t.vancutsem@ulg.ac.be
}

\begin{abstract}
Hybrid simulations combining phasor-mode and electromagnetic transients models aim at taking advantage of computational speed of the former and accuracy of the latter. For simulation accuracy, a relaxation process is used, which consists of iterating between both models. The convergence properties of this relaxation process are investigated. The speed of convergence is assessed when the variables exchanged at the interface between both models are respectively pure voltage/current sources, Norton/Thévenin equivalents, or a combination of them. Iteratively updated Norton/Thévenin equivalents are shown to drastically reduce the number of relaxation iterations.
\end{abstract}

Index Terms-Hybrid simulation, electromagnetic transients, phasor approximation, equivalents.

\section{INTRODUCTION}

$\mathbf{T}$ IME-domain simulation is used in power systems for many purposes, such as component design and sizing, dynamic security assessment, operator training, power quality assessment, etc. Dynamic phenomena are usually classified into two broad categories, according to the time constants of phenomena. Slower phenomena such as electromechanical transients, for instance, are traditionally studied with models derived under the Phasor Mode (PM) approximation, usually assuming positive-sequence voltages and currents. Faster phenomena falling in the category of ElectroMagnetic Transients (EMT) are studied with three-phase models. Mature software tools are available for both PM and EMT simulations.

Research efforts have been devoted towards taking advantage of the computational speed of PM simulations and the accuracy of EMT simulations, by combining them into a hybrid PM-EMT simulation [1]. To this purpose, the original power system is decomposed into two subsystems: one simulated under the PM approximation, referred to as the PM subsystem, and one represented with detailed EMT models, referred to as the EMT subsystem. Clearly, the former subsystem is to be simulated with a "large" time step size $H$, and the latter with a "small" step size $h$. A typical example of application is the detailed simulation of an unbalanced fault using the EMT model in a subsystem surrounding the fault location, and the PM model for the rest of the power system.

Significant advances have been made in hybrid PM-EMT simulation as testified by the state-of-the-art report in [2].

\footnotetext{
${ }^{a}$ Dept. of Elec. Eng. and Comp. Science, University of Liege, Belgium.

${ }^{b}$ Fund for Scientific Research (FNRS) at Dept. of Elec. Eng. and Comp. Science, University of Liege, Belgium.
}

However, there is still room for improvements to reach the targeted speed-accuracy compromise. The various points of possible improvement range from the theoretical foundations of this hybrid simulation [3] to practical issues such as the choice of the boundary between PM and EMT subsystems.

Clearly, the coupling of PM and EMT simulations implies representing the response of one subsystem when simulating the other subsystem. These cross-representations should be limited to simple "equivalents", for computational efficiency and implementation simplicity. Furthermore, by resorting to a relaxation scheme that iterates between the PM and EMT simulations, and by updating the equivalents from the results of the previous iteration, it is possible to compensate for their inaccuracy [4]. On the other hand, a proper choice of these equivalents may significantly speed up the convergence of the relaxation process.

A way to efficiently combine different simulation tools was considered in general terms in [5]. Although the hybrid PMEMT power system dynamic simulation is not considered specifically, this early publication shows the advantage of exchanging linear combinations of voltages and currents. It also proposes a methodology for analyzing the convergence, which has inspired the derivation reported in this paper. A study of the convergence of an iterative hybrid simulation scheme was also reported in [6]. This reference proposes to use an adaptive stabilizing factor to control both stability and speed of iterations.

In this paper the convergence properties of several equivalents are assessed. In particular it is shown that (reasonably accurate) Thévenin/Norton equivalents can improve the convergence of the relaxation process dramatically.

Section II describes the coupling of PM and EMT models in some more detail. Section III describes the simple test case subsequently used in Section IV, where the convergence of various equivalents is analyzed using an iteration factor inspired of the one used in [5]. The salient points are summarized in Section $\mathrm{V}$ by way of conclusion.

\section{Coupling The PM AND EMT MOdEls}

For simplicity, this paper focuses on the case where the PM and EMT subsystems are connected through a single bus.

The EMT and PM simulations must receive information compatible with their modeling assumptions. This re- 


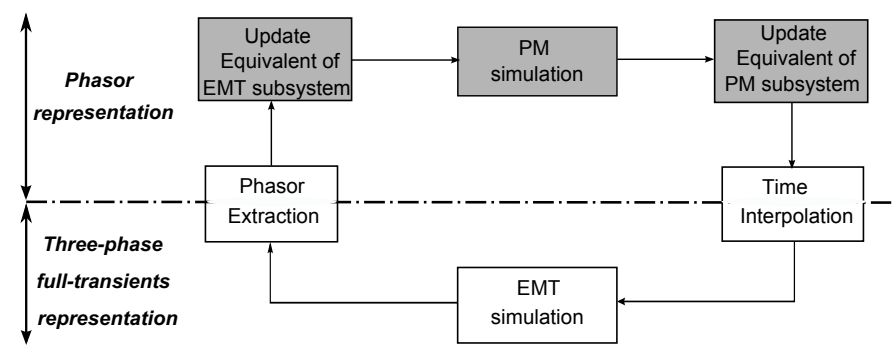

Figure 1. Overview of relaxation process

quires performing time interpolation and phasor extraction, as sketched in Fig. 1.

Time interpolation is needed to convert the "slowly" varying voltage and current phasors provided by the PM simulation into voltage and current sources evolving sinusoidally with time, at the nominal fundamental frequency, but with "slowly varying" magnitudes and phase angles.

Phasor extraction, used for passing information from EMT to PM simulation, consists of extracting the positive-sequence phasors from three-phase, bus voltage and branch current signals sampled at period $h$.

Further details can be found in [4], in particular about phasor extraction from distorted and unbalanced voltages and currents, which happens when the disturbance is located (in the EMT subsystem) too close to the boundary bus.

\section{A SIMPLE TEST SYSTEM}

A very simple test system has been used to validate the derivations, in particular under large disturbances and nonlinear effects not considered in the theoretical derivations of Section IV. This test system was chosen to focus on the convergence of different PM-EMT coupling schemes. Of course, it does not allow assessing the computational efficiency of the proposed hybrid simulation versus a full EMT simulation.

The one-line diagram is shown in Fig. 2. The PM subsystem only consists of a voltage source $\bar{E}_{p m}$ in series with an impedance $z_{p m}=j 0.01 \mathrm{pu}$ (on 100-MVA base). Bus 1 is the boundary. The EMT subsystem includes a 1,200-MVA roundrotor synchronous machine modeled with four rotor windings and a simple excitation and voltage regulation system. This subsystem has a three-phase, full-transients model of the EMTP type, involving 56 differential and 63 algebraic states.

A three-phase fault takes place on line 2-4. It is eliminated by opening the breakers at both ends of the line.

\section{CONVERGENCE PROPERTIES}

\section{A. Iteration factor}

A general formula for solving iteratively the simple scalar equation $a x=b$ is given by:

$$
x^{k+1}=w x^{k}+c \quad k=0,1,2, \ldots
$$

where all numbers are complex and $c=\frac{b}{a}(1-w)$ is a constant independent of $x^{k}$ and $x^{k+1} . w$ is called the iteration factor.

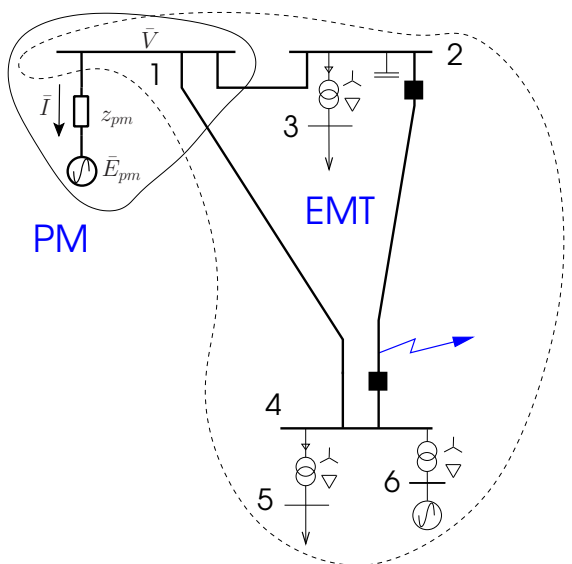

Figure 2. System used to test the convergence of the relaxation process
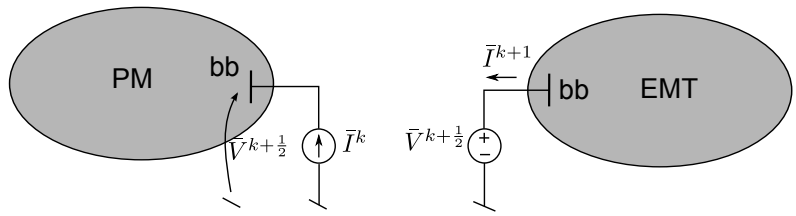

Figure 3. Zero-order boundary conditions ("bb" denotes the boundary bus, common to both PM and EMT subsystems)

The lower the norm of $w$, denoted $\|w\|$, the lower the number of iterations for the sequence $\left(x^{k}\right)$ to reach convergence, detected for instance from the norm $\| x^{k+1}-x^{k}||$ being smaller than some tolerance. If $\|w\|$ is much smaller than one, the convergence will take place rapidly, while if $\|w\|$ is larger than one, the sequence will not converge [7].

In what follows, various equivalents used in the PM-EMT relaxation process will be brought into the form (1).

\section{B. PM subsystem equivalenced by voltage source and EMT by current source}

The simplest equivalent, used in particular in [8], to model one subsystem when simulating the other is through voltage and current sources as shown in Fig. 3. Note that the phasor representation is used to comply with the PM model, but the equivalents are converted into time-varying sources and differential equations in the EMT simulation.

Let us consider the iterations performed when passing from time $t$ to time $t+H$, i.e. over one step of the PM simulation.

At a given iteration, the PM simulation (arbitrarily assumed to be carried out first) determines the evolution of the PM subsystem over a single step $H$, with the EMT subsystem replaced by a current source. The value $\bar{I}^{k}$ of the boundary current has been extracted from the EMT simulation performed at the previous iteration. This PM simulation yields the new estimate $\bar{V}^{k+\frac{1}{2}}$ of the boundary bus voltage, where the upperscript $k+\frac{1}{2}$ indicates that half of the iteration has been performed.

Next, using this voltage, the EMT simulation is carried out to obtain the EMT subsystem evolution from $t$ to $t+H$, using time steps $h$, and interpolating the evolution of the boundary bus voltage. This simulation yields the updated current $\bar{I}^{k+1}$.

Convergence is checked by comparing $\left\|\bar{V}^{k+1}-\bar{V}^{k}\right\|$ and $\left\|\bar{I}^{k+1}-\bar{I}^{k}\right\|$ to some tolerances. If the latter are satisfied, the 

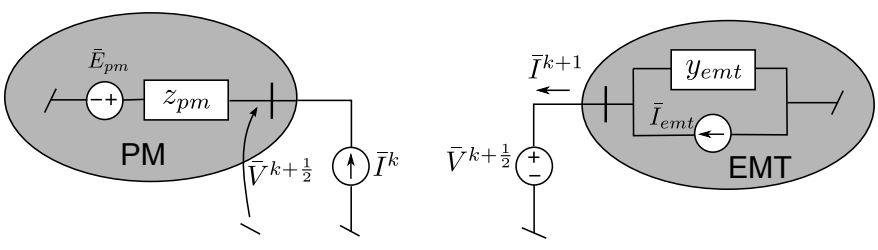

Figure 4. Configuration of Fig. 3 with the PM and EMT subsystems replaced by Thévenin and Norton equivalents

simulation proceeds with the next time interval $[t+H t+2 H]$. Otherwise, an additional relaxation iteration is performed.

In order to assess the speed of convergence, an approximation of the PM and EMT subsystems must be assumed. Let us suppose that, in the vicinity of the solution point, they can be linearized in respectively a Thévenin and a Norton equivalent, as shown in Fig. 4. From this figure, one easily obtains:

$$
\begin{aligned}
\bar{V}^{k+\frac{1}{2}} & =\bar{E}_{p m}+z_{p m} \bar{I}^{k} \\
\bar{I}^{k+1} & =\bar{I}_{e m t}-y_{e m t} \bar{V}^{k+\frac{1}{2}}
\end{aligned}
$$

Introducing (2) into (3) yields the recursive relation:

$$
\bar{I}^{k+1}=-z_{p m} y_{e m t} \bar{I}^{k}+\left(\bar{I}_{e m t}-y_{e m t} \bar{E}_{p m}\right)
$$

from which the iteration factor is easily identified as:

$$
w_{1}=-z_{p m} y_{e m t}
$$

Thus, for fast convergence, $\left\|w_{1}\right\|$ should be small. This is the case, for instance, if $z_{p m}$ tends to zero, i.e. if the PM subsystem behaves as a pure voltage source. In this case, its representation by the $\bar{V}^{k+\frac{1}{2}}$ voltage comes closer to its real behavior, and convergence will be fast. However, in all other cases (for instance if the PM subsystem behaves more like a current source) convergence is expected to be slow, or even divergence may take place. Similar considerations hold true for the EMT subsystem behaving as a current source.

This is illustrated in Fig. 5, obtained with the simple system of Section III. The case involves a fault applied to the EMT subsystem. The PM (resp. EMT) simulation uses a current (resp. voltage) source as shown in Fig. 3. The plot shows the successive values of the complex current (in Cartesian coordinates) during the relaxation process over the time step $H$ that follows the fault inception. It is easily seen that oscillations around the solution take place during the iterations. It takes as many as 64 iterations to converge, which clearly shows that zero-order boundary conditions do not yield satisfactory convergence of hybrid PM-EMT simulations. The value of the current to which the process converges is $\bar{I}=19.6124+j 7.3114 \mathrm{pu}$.

For the system of concern, replacing the generator by a constant e.m.f. behind subtransient reactance, the value of $y_{\text {emt }}$ computed from the reduced nodal admittance matrix is $y_{e m t}^{\star}=10.4083-j 88.8711 \mathrm{pu}$. This leads to $w_{1}=$ $-0.8887+j 0.1041$. The magnitude $\left\|w_{1}\right\|=0.8948$, being close to unity, explains the slow convergence. Also, $w_{1}$ is close to a real negative number, which explains the oscillatory behavior of the successive iterates around the solution.

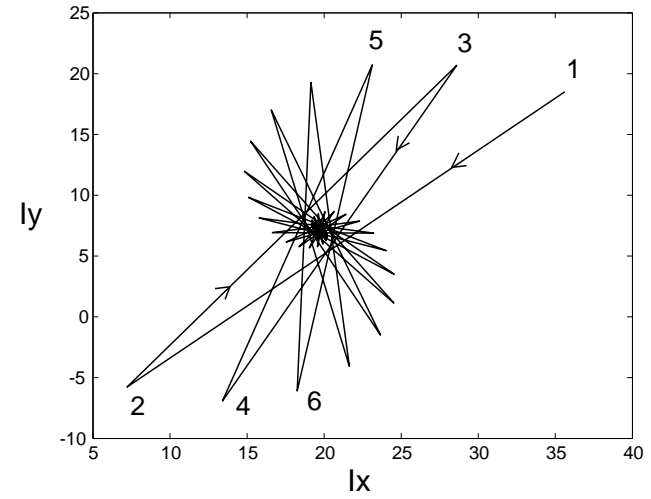

Figure 5. Example of iterations performed with the equivalents of Fig. 3. The first six iterations are identified with numbers

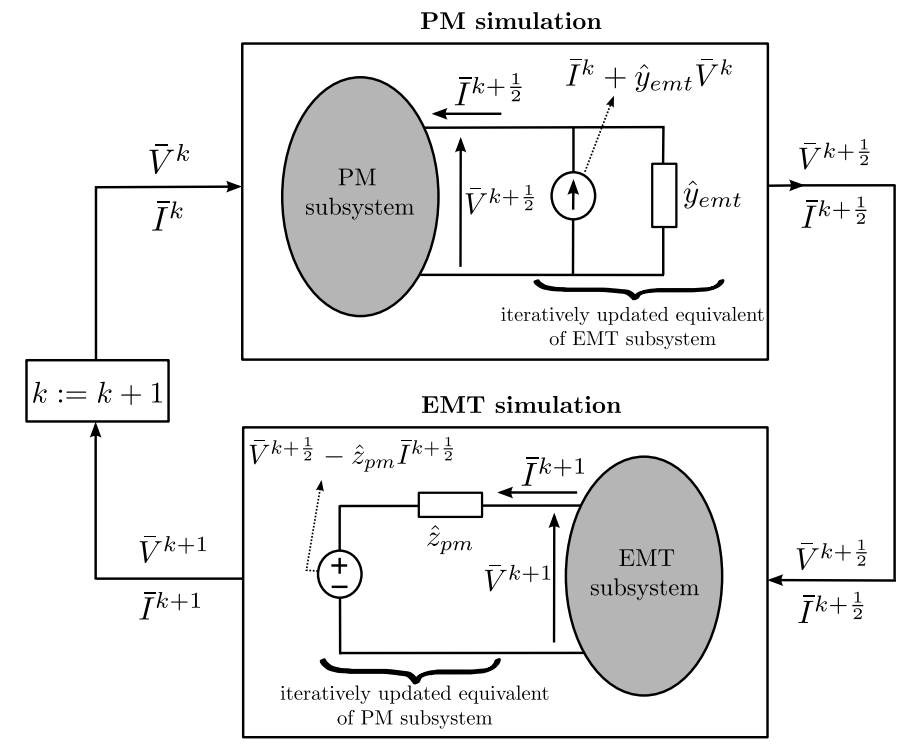

Figure 6. First-order boundary conditions

\section{PM subsystem equivalenced by Thévenin and EMT by} Norton

The equivalents used in Fig. 3 can be referred to as "zeroorder boundary conditions" because, at the boundary between both subsystems, the current (resp. voltage) source is constant when performing the PM (resp. the EMT) simulation. It does not depend on the voltage (current) being computed by the PM (resp. EMT) simulation, and will be updated only at the next iteration of the relaxation process.

First-order boundary conditions, involving a linear relation between voltage and current are now considered. They consist of Thévenin and Norton equivalents as shown in Fig. 6. This configuration was proposed in [9] and is also used in [4]. Note that Norton or Thévenin equivalents can be used interchangeably.

The PM simulation is performed with EMT subsystem replaced by a Norton equivalent, involving an admittance $\hat{y}_{e m t}$ and the Norton current $\bar{I}^{k}+\hat{y}_{e m t} \bar{V}^{k}$ updated with the latest available boundary voltage and current. In case a fault 
is simulated in the EMT subsystem, $\hat{y}_{\text {emt }}$ can be computed beforehand for the pre-fault, during-fault and post-fault situations. Alternatively, it could be estimated numerically, from the previous values of the boundary voltage and current.

Similarly, the EMT simulation is performed with the PM subsystem replaced by a Thévenin equivalent, involving an impedance $\hat{z}_{p m}$ (also determined beforehand or numerically), and the Thévenin voltage $\bar{V}^{k+\frac{1}{2}}-\hat{z}_{p m} \bar{I}^{k+\frac{1}{2}}$ also updated with the latest available boundary voltage and current.

The following relations are easily derived from Fig. 6:

$$
\begin{aligned}
& \bar{I}^{k+\frac{1}{2}}=\bar{I}^{k}+\hat{y}_{e m t} \bar{V}^{k}-\hat{y}_{e m t} \bar{V}^{k+\frac{1}{2}} \\
& \bar{V}^{k+1}=\bar{V}^{k+\frac{1}{2}}-\hat{z}_{p m} \bar{I}^{k+\frac{1}{2}}+\hat{z}_{p m} \bar{I}^{k+1}
\end{aligned}
$$

As for the zero-order boundary conditions, we assume at this point that the PM and EMT subsystems can be linearized in respectively a Thévenin and a Norton equivalent, as shown in Fig. 4, with the following equations:

$$
\begin{aligned}
\bar{V}^{k+\frac{1}{2}} & =\bar{E}_{p m}+z_{p m} \bar{I}^{k+\frac{1}{2}} \\
\bar{I}^{k+1} & =\bar{I}_{e m t}-y_{e m t} \bar{V}^{k+1}
\end{aligned}
$$

From Eqs. (6 - 9), the iteration factor is obtained as:

$$
w_{2}=\underbrace{-z_{p m} y_{e m t}}_{w_{1}} \frac{1-\frac{\hat{z}_{p m}}{z_{p m}}}{1+\hat{z}_{p m} y_{e m t}} \frac{1-\frac{\hat{y}_{e m t}}{y_{e m t}}}{1+z_{p m} \hat{y}_{e m t}}
$$

This result shows that $\hat{z}_{p m}$ and $\hat{y}_{e m t}$ can be used as degrees of freedom to make the iteration factor $w_{2}$ smaller in magnitude than $w_{1}$. More precisely, the iteration factor $w_{2}$ can be decreased by choosing $\hat{z}_{p m} \simeq z_{p m}$, i.e. by using a Thévenin impedance $\hat{z}_{p m}$ close to the exact value $z_{p m}$. It can also be decreased by choosing $\hat{y}_{e m t} \simeq y_{e m t}$, i.e. by using a Norton admittance $\hat{y}_{e m t}$ close to $y_{e m t}$.

\section{PM subsystem equivalenced by Thévenin and EMT by current source}

Variants combining voltage/current sources with Thévenin/Norton equivalents (i.e. mixed zero- and firstorder boundary conditions) can be also contemplated. In Fig. 7, for instance, the PM subsystem is represented by a Thévenin equivalent in the EMT simulation, while the EMT subsystem is represented by a current source in the PM simulation. Formally, this corresponds to setting $\hat{y}_{e m t}$ to zero, and involving $\bar{I}^{k}$ instead of $\bar{I}^{k+\frac{1}{2}}$ in the Thévenin equivalent (indeed, the current injected in the PM subsystem is not updated by the PM simulation). This configuration (or improved variants of the latter) is used in [10]-[13].

Through derivations similar to the ones in Sections IV-B and IV-C the following iteration factor is found:

$$
w_{3}=\underbrace{-z_{p m} y_{e m t}}_{w_{1}} \frac{1-\frac{\hat{z}_{p m}}{z_{p m}}}{1+\hat{z}_{p m} y_{e m t}}
$$

No result is given for this configuration since the PM part of the test system is already modeled by a simple Thévenin equivalent (see Fig. 2).

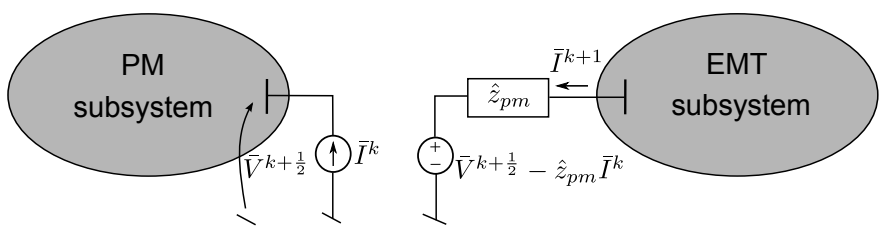

Figure 7. Mixed zero- and first-order boundary conditions - first variant

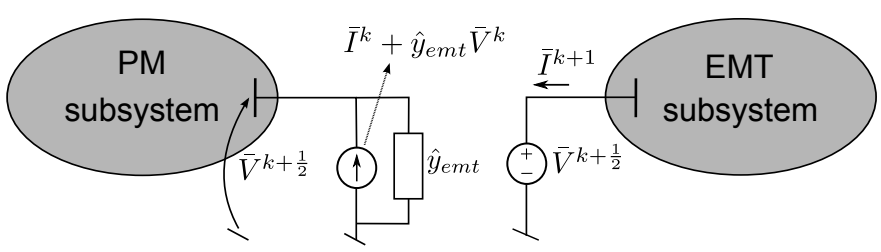

Figure 8. Mixed zero- and first-order boundary conditions - second variant

E. PM subsystem equivalenced by voltage source and EMT by Norton

The last variant is shown in Fig. 8, with the PM subsystem represented by a voltage source in the EMT simulation, and the EMT subsystem by a Norton equivalent in the PM simulation. Formally, this corresponds to setting $\hat{z}_{p m}$ to zero.

To the authors' knowledge, this configuration has not been considered in the literature. The reason is that most publications (e.g. [13]) consider the possibility for the PM-EMT subsystem boundary to be close to the disturbance location, and use a shunt admittance to approximate the response of the PM subsystem to harmonics generated by the EMT model.

The following iteration factor is obtained:

$$
w_{4}=\underbrace{-z_{p m} y_{e m t}}_{w_{1}} \frac{1-\frac{\hat{y}_{e m t}}{y_{e m t}}}{1+z_{p m} \hat{y}_{e m t}}
$$

To assess the impact of using an estimated Norton admittance $\hat{y}_{e m t}$ that significantly departs from the exact (but unknown) value $y_{e m t}$, Fig. 9 shows the evolution of $\left\|w_{4}\right\|$ with $\left\|\frac{\hat{y}_{\text {emt }}}{y_{\text {emt }}}\right\|$, for two values of the product $z_{p m} y_{e m t}$. Note the logarithmic scale used in abscissa. This plot suggests that $\left\|w_{4}\right\|$ remains small (i.e. convergence remains fast) even when $\hat{y}_{e m t}$ significantly departs from $y_{\text {emt }}$. Furthermore, when $z_{p m} y_{e m t}=10,\left\|w_{4}\right\|$ remains reasonably small, while $w_{1}=-z_{p m} y_{e m t}=-10$, indicating that the equivalent of Fig. 3 would lead to severely diverging iterations.

Getting back to the system of Section III, the scenario already considered in Fig. 5 has been simulated with the equivalent of Fig. 8. Fig. 10 shows the successive values of the complex current (in Cartesian coordinates) during the relaxation process over the time step $H$ that follows the fault inception. It takes only three iterations for the relaxation process to converge. Compared to Fig. 5, the number of iterations is drastically reduced when using a first-order boundary condition, which is thus recommended for hybrid PM-EMT simulation. Furthermore the final value of the current is found to be $\bar{I}=19.6128+j 7.3115 \mathrm{pu}$, i.e. the same value as in Fig. 5. 


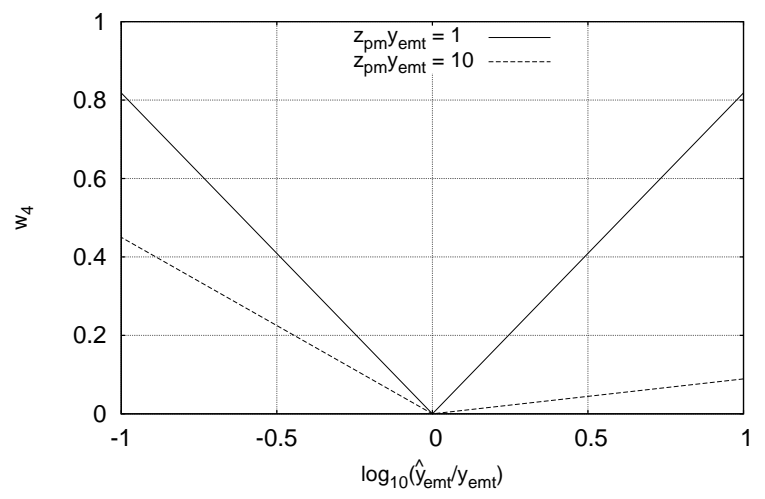

Figure 9. Variation of $\left\|w_{4}\right\|$ with $\left\|\frac{\hat{y}_{e m t}}{y_{e m t}}\right\|$ for two values of $z_{p m} y_{e m t}$

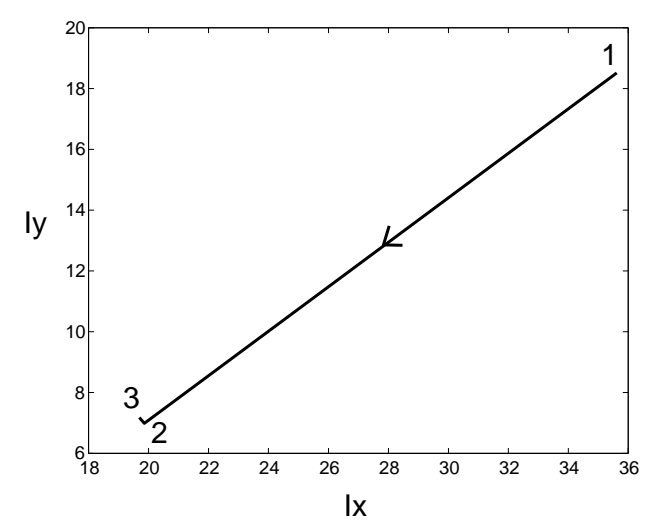

Figure 10. Example of iterations performed with the equivalents of Fig. 8

The results of Fig. 10 have been obtained with $\hat{y}_{e m t}=y_{e m t}^{\star}$, the Norton admittance estimated from a simple linear approximation of the EMT subsystem (see Section IV-B). Figure 11 shows the variation of the number of relaxation iterations for various values of $\hat{y}_{\text {emt }}$ obtained by multiplying $\hat{y}_{\text {emt }}^{\star}$ by a real, positive number. The number of iterations is the lowest for $\hat{y}_{e m t}=y_{e m t}^{\star}$. The shape of the curve is remarkably close to the one of Fig. 9.

\section{CONCLUSION}

Phasor-mode and electromagnetic transient simulations can be combined through a relaxation process which, at each time step of the phasor-mode simulation, iterates between the two models until convergence is reached. This requires using an iteratively updated equivalent of one subsystem when simulating the other one. This paper has considered the impact of such equivalents on the speed of convergence of the relaxation process. It has been shown that the speed can be significantly improved provided one of the two equivalents takes into account the linear dependency of the boundary bus voltage with the boundary current, in the form of either a Thévenin or a Norton equivalent. The fast convergence paves the way to real-time simulation applications where only one iteration of the above relaxation process would be performed.

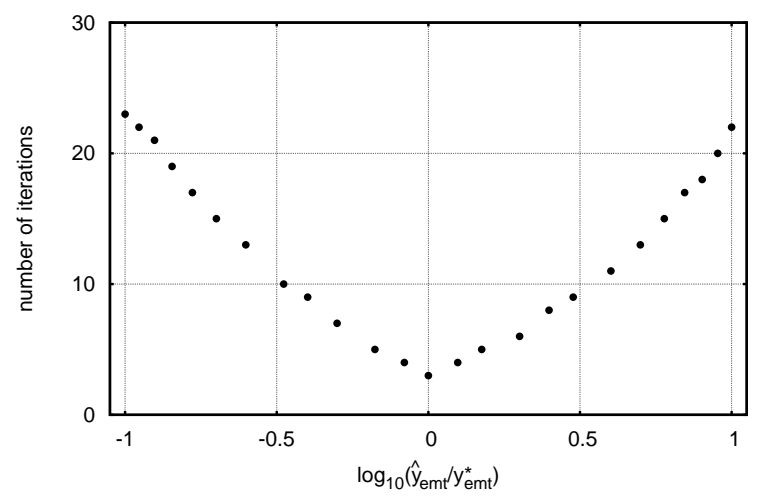

Figure 11. Number of relaxation iterations for various values of $\frac{\hat{y}_{e m t}}{y_{\text {emt }}^{\star}}$

\section{ACKNOWLEDGMENT}

Work supported by Belgian Science Policy (IAP P7/02) and Walloon Region of Belgium (WBGreen Grant FEDO).

\section{REFERENCES}

[1] M. Heffernan, K. Turner, J. Arrillaga, and C. Arnold, "Computation of ac-dc system disturbances - part i, ii and iii. interactive coordination of generator and converter transient models," IEEE Transactions on Power Apparatus and Systems, vol. 100, no. 11, pp. 4341 - 4363, 1981.

[2] V. Jalili-Marandi, V. Dinavahi, K. Strunz, J. Martinez, and A. Ramirez, "Interfacing techniques for transient stability and electromagnetic transient programs," IEEE Transactions on Power Delivery, vol. 24, no. 4, pp. $2385-2395,2009$.

[3] S. Fan and H. Ding, "Time domain transformation method for accelerating emtp simulation of power system dynamics," IEEE Transactions on Power Systems, vol. 27, pp. 1778-1787, 2012.

[4] F. J. Plumier, P. Aristidou, C. Geuzaine, and T. Van Cutsem, "A relaxation scheme to combine phasor-mode and electromagnetic transients simulations," paper submitted for presentation at the 18th Power System Computation Conference, Wroclaw, Aug 2014.

[5] V. B. Dmitriev-Zdorov and B. Klaassen, "An improved relaxation approach for mixed system analysis with several simulation tools." in EURO-DAC. IEEE Computer Society, 1995, pp. 274-279.

[6] B. Kasztenny and M. Kezunovic, "A method for linking different modeling techniques for accurate and efficient simulation," IEEE Transactions on Power Systems, vol. 15, no. 1, pp. 65 - 72, 2000.

[7] J. K. White and A. Sangiovanni-Vincentelli, Relaxation Techniques for the Simulation of VLSI Circuits. Kluwer Academic Publishers, 1987.

[8] F. Plumier, C. Geuzaine, and T. Van Cutsem, "A multirate approach to combine electromagnetic transients and fundamental-frequency simulations," in Proceedings of International Conference on Power System Transients (IPST), 2013.

[9] G. W. Anderson, "Hybrid simulation of ac-dc power systems," Ph.D. dissertation, University of Canterbury, Christchurch, New Zealand, 1995.

[10] W. zhuo Liu, J. xian Hou, Y. Tang, L. Wan, X. li Song, and S. tao Fan, "An electromechanical/electromagnetic transient hybrid simulation method that considers asymmetric faults in an electromechanical network," in Proceedings of the Power Systems conference and exposition (PSCE), Phoenix, USA, March 20-23 2011.

[11] A. van der Meer, R. Hendriks, M. Gibescu, and W. Kling, "Interfacing methods for combined stability and electro-magnetic transient simulations applied to vsc-hvdc," in Proceedings of International Conference on Power System Transients (IPST), Delft, The Netherlands, 2011.

[12] S. Abhyankar and A. Flueck, "Parallel-in-space-and-time scheme for implicitly coupled electromechanical and electromagnetic transients simulation," in Proceedings of International Conference on Power System Transients (IPST), 2013.

[13] Y. Zhang, A. Gole, W. Wu, B. Zhang, and H. Sun, "Development and analysis of applicability of a hybrid transient simulation platform combining tsa and emt elements," IEEE Transactions on Power Systems, vol. 28, no. 1, pp. 357-366, 2013. 